2 Q33 DUWDO+ HGJ LQJIDQG \& RXQAHUP RQRIRQAF6 XP V

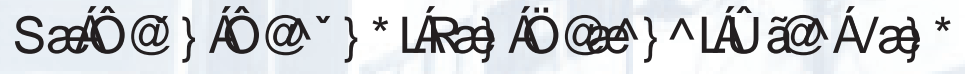

DEPARTMENT OF ACCOUNTANCY, FINANCE AND INSURANCE (AFI) 


\title{
On Partial Hedging and Counter-monotonic Sums
}

\author{
Ka Chun Cheung Jan Dhaene Qihe Tang
}

August 4, 2011

\begin{abstract}
In this article, we show, in the context of partial hedging, that some important relationships about comonotonicity and convex order cannot be translated to countermonotonicity in general because of the possibility of over-hedging. We propose a new notion called proper hedge that can effectively avoid over-hedging. Different characterizations of a proper hedge are given, and we show that this notion is useful in translating relationship between comonotonicity and convex order to the case of counter-comonotonicity. As an application, we apply our results to identify desirable structural properties of insurance indemnities that make an insurance contract appealing to both the policyholder and the insurer.

Key words: hedging, comonotonicity, counter-monotonicity, convex order, Tail Valueat-Risk
\end{abstract}

\section{Introduction}

Consider a portfolio with random value $X$ at the end of the reference period. We define a hedge for this portfolio as an additional asset that can be added to the portfolio to offset the price movements of the original portfolio. Hence, adding the hedge reduces the risk (in some sense) associated with the portfolio value at the end of the reference period. To formalize the idea, we introduce the following definition.

Definition 1 A random variable $Z$ is said to be a hedge for the random variable $X$ if

$$
\operatorname{Var}(X+Z) \leq \operatorname{Var}(X) .
$$

Moreover, $Z$ is said to be a convex hedge for $X$ if

$$
X+Z \leq_{c x} X+\mathbb{E}(Z) .
$$

If, in addition $(X, Z)$ is counter-monotonic, $Z$ is said to be a counter-monotonic convex hedge for $X$. 
Obviously, a convex hedge is a hedge, but not vice versa in general.

In mathematical finance or in portfolio theory, a hedge usually refers to a self-financing portfolio that replicates some given financial claim at a future time point, see, for instance, Björk (2009). In this paper, the definition of a (convex, counter-monotonic convex) hedge is less stringent. The requirement of exact replication is relaxed to reduction of risk (variability) defined through variance order or convex order. Therefore, the (convex, counter-monotonic convex) hedges we define and study in this paper have to be understood as partial hedges in the traditional sense.

Before investigating the notion of counter-monotonic convex hedges, we recall the following well-known properties about comonotonic sums and convex order. For any integrable random variables $X, Y$, and $Z$,

$$
X \leq_{c x} Y, \quad(Y, Z) \text { is comonotonic } \quad \Longrightarrow \quad X+\mathbb{E}(Z) \leq_{c x} Y+Z
$$

and

$$
X \leq_{c x} Y, \quad(Y, Z) \text { is comonotonic } \Longrightarrow X+Z \leq_{c x} Y+Z .
$$

Both (2) and (3) are special cases of a more general result (see Lemma 4 below), which asserts that if $X_{i} \leq_{c x} Y_{i}$ for $i=1,2$ and if $\left(Y_{1}, Y_{2}\right)$ is comonotonic, then $X_{1}+X_{2} \leq_{c x} Y_{1}+Y_{2}$.

Since comonotonicity and counter-monotonicity are two opposite extremal dependence structures, it is natural to consider whether analogous results of (2) and (3) hold for countermonotonicity. More precisely, one conjectures that

$$
X \leq_{c x} Y, \quad(X, Z) \text { is counter-monotonic } \quad \Longrightarrow \quad X+Z \leq_{c x} Y+\mathbb{E}(Z) .
$$

and

$$
X \leq_{c x} Y, \quad(X, Z) \text { is counter-monotonic } \quad \Longrightarrow \quad X+Z \leq_{c x} Y+Z
$$

One possible interpretation of statement (4) is that if position $X$ is less risky than position $Y\left(X \leq_{c x} Y\right)$, and asset $Z$ can offset price movements of $X((X, Z)$ is counter-monotonic), then position $X+Z$ is again less risky than $Y$ (plus the constant $\mathbb{E}(Z)$ ). In particular, according to Definition 1, (4) would mean that any asset that is counter-monotonic with $X$ is a convex hedge of $X$. For statement (5), the interpretation would be that the order of riskiness is preserved on the portfolio level when an asset that is counter-comonotonic to the less risky sub-portfolio is added to both sub-portfolios.

Unfortunately, neither conjecture (4) nor (5) holds true in general, as demonstrated by the following counter-example.

Example 1 Let $W$ be any standard normal variable, and define

$$
(X, Y, Z):=(-W / 2,-W, 2 W)
$$


then $X \leq_{c x} Y$, and $(X, Z)$ is counter-monotonic. But $X+Z=3 W / 2 \geq_{c x}-W=Y$, which contradicts (4). If we define

$$
(X, Y, Z):=(-W / 2,-W, W)
$$

instead, then $X+Z=W / 2 \geq_{c x} 0=Y+Z$, which contradicts (5).

The reason why $X+Z$ is even riskier than both $Y$ and $Y+Z$ in the example above is that $Z$ "over-hedges" $X$ in that adding $Z$ to $X$ not only eliminates the original risk of $X$ but also introduces additional risk. Indeed, we can easily see that counter-monotonicity of $(X, Z)$ does not necessarily imply that $Z$ is a hedge for $X$. To this end, we notice that

$$
\operatorname{Var}(X+Z) \leq \operatorname{Var}(X) \Longleftrightarrow \operatorname{Var}(Z) \leq-2 \operatorname{Cov}(X, Z)
$$

The counter-monotonicity of $(X, Z)$ only guarantees the positivity of $-2 \mathrm{Cov}(X, Z)$, but in case $\operatorname{Var}(Z)$ is sufficiently large when compared to $-2 \operatorname{Cov}(X, Z), Z$ will not be a hedge, and thus not a convex hedge for $X$. This simple consideration suggests the possibility of over-hedging if $Z$ itself is too risky.

From the above counter-example and discussion, one sees that for a given position $X$, not every counter-monotonic asset $Z$ is risk-reducing because of the possibility of over-hedging. The objectives of this paper are twofold. First, in Section 3, we propose a new notion called proper hedge, which requires a counter-monotonic asset to satisfy an additional condition. Natural interpretations and different characterizations of the additional condition will be given. In Section 4, we prove that a proper hedge will never over-hedge by showing that both conjectures (4) and (5) hold true when $Z$ is a proper hedge of $X$. In Section 5, we apply results obtained in Sections 3 and 4 to the study of the marketability of insurance indemnities. We identify desirable properties that an indemnity schedule should possess in order for the insurance contracts to be beneficial to both the policyholder and the insurer. Finally, in Section 6, we extend and complement various existing results and results established in Sections 3 and 4 by considering more general dependence structures such as (positive and negative) association and quadrant dependence.

Before closing this introduction, we want to emphasize that the concepts and definitions of hedging studied in this paper are purely based on stochastic ordering in which the level of riskiness of positions are compared by convex order. We do not take into consideration the preference of individual decision maker as well as the (no-arbitrage) market price of a hedge. Indeed, while a convex hedge can reduce the risk of a given position, an expected utility maximizer is not always better off by buying a convex hedge. To see this, assume that his utility function is given by some increasing and concave function $u$. He will only purchase an additional asset $Z$ if the following inequality is fulfilled:

$$
\mathbb{E}([u(X+Z-\pi(Z))] \geq \mathbb{E}([u(X)],
$$

where $\pi(Z)$ is the market price of $Z$. The fact that $Z$ is a convex hedge for $X$ does not necessarily imply that (6) is fulfilled. Furthermore, it may happen that inequality (6) is fulfilled even if $Z$ is not a convex hedge for $X$. These possibilities are illustrated by the following example. 
Example 2 (i) Suppose that $Z$ is a convex hedge for $X$ (so that $X+Z \leq_{c x} X+\mathbb{E}(Z)$ by definition). If $\pi(Z)>\mathbb{E}(Z)$ and $u(x):=x$ for all $x$, then (6) is violated.

(ii) Suppose that $Z$ is a random variable such that $\operatorname{Var}(Z)>-2 \operatorname{Cov}(X, Z)$. If $\pi(Z)=\mathbb{E}(Z)$ and $u(x)=x$ for all $x$, then (6) is fulfilled, but $Z$ is not a convex hedge of $X$ because (1) is violated.

In spite of these negative results, we will show in Section 5 that the idea of convex hedges and the results to be established in Sections 3 and 4 have an interesting application in the context of designing insurance indemnity schedules, in which the decision maker is assumed to be an expected utility maximizer.

\section{Preliminaries}

This section serves to introduce the notations used in this paper and recall the definitions and properties of three inter-related concepts, namely, convex order, comonotonicity, and Tail Value-at-Risk (TVaR).

All random variables discussed in this paper are defined on a common probability space $(\Omega, \mathcal{F}, \mathbb{P})$, and are assumed to be integrable. Given random variables $X$ and $Y$, we say that $X$ is smaller than $Y$ in the stop-loss order (written as $\left.X \leq_{s l} Y\right)$ if $\mathbb{E}(X-d)_{+} \leq \mathbb{E}(Y-d)_{+}$ for all real numbers $d$. If, in addition, $\mathbb{E}(X)=\mathbb{E}(Y)$, then we say that $X$ is smaller than $Y$ in the convex order (written as $X \leq_{c x} Y$ ). It can be shown that $X \leq_{c x} Y$ if and only if $\mathbb{E}[f(X)] \leq \mathbb{E}[f(Y)]$ for any convex function $f$ for which the two expectations exist. We refer to Denuit et al. (2005) and Shaked and Shanthikumar (1994) for a comprehensive treatment of convex order.

A useful characterization of convex order using TVaR is given by the next lemma. Throughout the paper, for a distribution function $F$, we use $F^{-1}$ to denote its left-continuous inverse:

$$
F^{-1}(p):=\inf \{x \in \mathbb{R} \mid F(x) \geq p\}, \quad 0<p<1 .
$$

For a random variable $Y$, its TVaR at the probability level $p$ is defined as

$$
\operatorname{TVaR}_{p}(Y):=\frac{1}{1-p} \int_{p}^{1} F_{Y}^{-1}(t) d t, \quad 0<p<1 .
$$

Lemma $1 X \leq_{c x} Y$ if and only if $\mathbb{E}(X)=\mathbb{E}(Y)$ and $T \operatorname{Va} R_{p}(X) \leq T \operatorname{Ta} R_{p}(Y)$ for all $0<p<1$.

Lemma 2 TVaR is sudadditive: For any random variables $X$ and $Y$,

$$
\operatorname{TVaR}_{p}(X+Y) \leq \operatorname{TVaR}_{p}(X)+\operatorname{TVaR}_{p}(Y) \text { for all } 0<p<1 \text {. }
$$


For proofs of these two lemmas and other properties of TVaR, we refer to Dhaene et al. (2006).

A random vector $\left(Y_{1}, \ldots, Y_{n}\right)$ is comonotonic if there exist a random variable $V$ and $n$ increasing functions $g_{1}, \ldots, g_{n}$ such that

$$
\left(Y_{1}, \ldots, Y_{n}\right) \stackrel{d}{=}\left(g_{1}(V), \ldots, g_{n}(V)\right) .
$$

In particular, we may choose $V$ to be any uniform $(0,1)$ random variable, and $g_{i}$ to be $F_{Y_{i}}^{-1}$. Furthermore, by Corollary 6.11 of Kallenberg $(2002)$, there is a uniform $(0,1)$ random variable $U$ such that an apparent stronger condition holds true:

$$
\left(Y_{1}, \ldots, Y_{n}\right)=\left(F_{Y_{1}}^{-1}(U), \ldots, F_{Y_{n}}^{-1}(U)\right) \text { almost surely. }
$$

Equivalently, $\left(Y_{1}, \ldots, Y_{n}\right)$ is comonotonic if there is a null set $N$ such that for any $i, j \in$ $\{1,2, \ldots, n\}$,

$$
\left(Y_{i}(\omega)-Y_{i}\left(\omega^{\prime}\right)\right)\left(Y_{j}(\omega)-Y_{j}\left(\omega^{\prime}\right)\right) \geq 0, \quad \omega, \omega^{\prime} \in \Omega \backslash N .
$$

A pair of random variables $(X, Y)$ is said to be counter-monotonic if $(X,-Y)$ is comonotonic.

Lemma $3(X, Y)$ is comonotonic if and only if

$$
\operatorname{TVaR}_{p}(X+Y)=\operatorname{TVaR}_{p}(X)+\operatorname{TVaR}_{p}(Y) \text { for all } 0<p<1 \text {. }
$$

The "only if" is a well-known result (see Dhaene et al. (2006)). The proof of the "if" part can be found in Cheung (2010).

The next two lemmas summarize some fundamental relationships between comonotonicity and the convex order. For proofs and further discussion, we refer to Dhaene et al. (2002a, 2002b).

Lemma 4 If $X_{i} \leq_{c x} Y_{i}$ for $i=1,2$ and if $\left(Y_{1}, Y_{2}\right)$ is comonotonic, then

$$
X_{1}+X_{2} \leq_{c x} Y_{1}+Y_{2}
$$

The next lemma is taken from Dhaene and Goovaerts (1996).

Lemma 5 If $(X, Y)$ and $\left(X^{c c}, Y^{c c}\right)$ have the same marginal distributions and the latter is counter-monotonic, then

$$
X^{c c}+Y^{c c} \leq_{c x} X+Y .
$$




\section{Proper hedges}

We saw that conjectures (4) and (5) fail to hold true because the counter-monotonic asset $Z$ may over-hedge the original position $X$. In this section, we suggest one extra condition to be imposed on a counter-monotonic asset so that over-hedging will not arise. To motivate our discussion, we first consider the following simple example.

Example 3 Suppose that we are holding a unit of stock. Let us denote the price of the stock at a future time point $T$ by $X$. A common strategy to reduce the risk of this position is to purchase a European put option on this stock. After adding $Z:=(K-X)_{+}$to $X$, the new position is given by $X+Z=\max (X, K)$. Obviously, it is less risky than $X$. Another strategy is to short one share of the same stock. In this case, $Z:=-X$ and thus the new position $X+Z$ is identically zero. On the other hand, it is generally believed that longing two European put options on the stock is not a good strategy to adopt, because in this case it is not clear whether the new position $X+2(K-X)_{+}$is less risky than $X$.

While all of the three strategies (holding one put, shorting one unit of stock, and holding two puts) considered in Example 3 consist of adding counter-monotonic assets, our intuition suggests that the first two will not over-hedge but not necessarily the third one. There are two different heuristic considerations that allow us to distinguish the first two strategies from the third:

(1) In the first two strategies (holding a put or shorting a unit of stock), the hedged position $X+Z$ preserves the nature of the original position $X$ in that both of them are bullish on the stock price. In other words, $(X, X+Z)$ is comonotonic, and hence the belief in how the markets will move remains unchanged after $Z$ is added. If $X$ performs well, so does the hedged position $X+Z$. However, the variation of the latter position (after $Z$ is added) is smaller because of the offsetting nature of $Z$. For the third strategy (holding two puts), the position $X+Z$ is no longer comonotonic with $X$.

(2) While $Z$ and $X$ move in opposite directions, the decrement (or increment) of $Z$ is always less than the increment (or decrement) of $X$ in the first two strategies. In this way, adding $Z$ to $X$ will not bring in an extra and unwanted price fluctuation. However, the decrement (or increment) of $Z$ is always larger than the increment (or decrement) of $X$ in the third strategy.

To sum up, in order to avoid a counter-monotonic position $Z$ to over-hedge $X$, the first consideration suggests that $(X, X+Z)$ has to be comonotonic, while the second consideration suggests that $Z$ should have a smaller fluctuation when compared to $X$. In the next result, we show that these two considerations are indeed equivalent.

Lemma 6 If $(X, Z)$ is counter-monotonic, then the following statements are equivalent: 
(a) $(X, X+Z)$ is comonotonic;

(b) $(Z, X+Z)$ is counter-monotonic;

(c) for some null set $N$,

$$
\left|Z(\omega)-Z\left(\omega^{\prime}\right)\right| \leq\left|X(\omega)-X\left(\omega^{\prime}\right)\right| \quad \text { for any } \omega, \omega^{\prime} \in \Omega \backslash N .
$$

Proof: The equivalence between (a) and (b) is obvious.

Assume that (c) is true. Fix some $\omega, \omega^{\prime} \in \Omega \backslash N$ and assume that $X\left(\omega^{\prime}\right) \leq X(\omega)$. Then

$$
\left(X(\omega)-X\left(\omega^{\prime}\right)\right)\left(X(\omega)+Z(\omega)-X\left(\omega^{\prime}\right)-Z\left(\omega^{\prime}\right)\right) \geq 0,
$$

which means that $(X, X+Z)$ is comonotonic. Therefore, (a) is true.

Now we assume that (a) holds true. Then there is a uniform $(0,1)$ random variable $U$ such that

$$
(X, X+Z,-Z)=\left(F_{X}^{-1}(U), F_{X+Z}^{-1}(U), F_{-Z}^{-1}(U)\right)
$$

almost surely. Denote by $N$ the corresponding null set. Outside this null set, we have

$$
F_{X}^{-1}(U)-F_{X+Z}^{-1}(U)=X-(X+Z)=-Z=F_{-Z}^{-1}(U) .
$$

Therefore,

$$
F_{X}^{-1}(\cdot)-F_{-Z}^{-1}(\cdot)=F_{X+Z}^{-1}(\cdot)
$$

almost everywhere (with respect to the Lebesgue measure) on $(0,1)$. Suppose that this equality fails to hold true for some $u^{*} \in(0,1)$, say $F_{X}^{-1}\left(u^{*}\right)-F_{-Z}^{-1}\left(u^{*}\right)<F_{X+Z}^{-1}\left(u^{*}\right)$. By the left-continuity of inverse distribution functions, there exists some $\varepsilon>0$ such that $F_{X}^{-1}(u)-F_{-Z}^{-1}(u)<F_{X+Z}^{-1}(u)$ for all $u \in\left[u^{*}-\varepsilon, u^{*}\right]$, which leads to a contradiction. Therefore, the equality $F_{X}^{-1}-F_{-Z}^{-1}=$ $F_{X+Z}^{-1}(\cdot)$ actually holds on the whole unit interval. From this, we obtain that $F_{X}^{-1}-F_{-Z}^{-1}$ is an increasing function.

For any $\omega, \omega^{\prime}$ outside $N$, assume without losing generality that $0<U\left(\omega^{\prime}\right)<U(\omega)<1$. Then

$$
F_{X}^{-1}\left(U\left(\omega^{\prime}\right)\right)-F_{-Z}^{-1}\left(U\left(\omega^{\prime}\right)\right) \leq F_{X}^{-1}(U(\omega))-F_{-Z}^{-1}(U(\omega))
$$

and hence

$$
\left|F_{-Z}^{-1}(U(\omega))-F_{-Z}^{-1}\left(U\left(\omega^{\prime}\right)\right)\right| \leq\left|F_{X}^{-1}(U(\omega))-F_{X}^{-1}\left(U\left(\omega^{\prime}\right)\right)\right|,
$$

from which (c) follows.

Motivated by the above discussion, we introduce the following definition.

Definition 2 A position $Z$ is said to be a proper hedge of $X$ if

(i) $(X, Z)$ is counter-monotonic, and 
(ii) $Z$ satisfies any one of the equivalent conditions stated in Lemma 6.

The collection of all proper hedges of $X$ is denoted as $H(X)$.

The next result shows that the counter-comonotonicity of $(Z, X+Z)$ (condition (b) of Lemma 6 ) implicitly implies the counter-monotonicity of $(X, Z)$, and hence this condition alone is equivalent to $Z \in H(X)$.

Lemma $7 Z \in H(X)$ if and only if $(Z, X+Z)$ is counter-comonotonic.

Proof: Suppose that $Z \in H(X)$. By definition and Lemma $6,(X, Z)$ is counter-monotonic, and $(X, X+Z)$ is comonotonic. Therefore, $(-Z, X+Z)$ is comonotonic.

Conversely, suppose that $(-Z, X+Z)$ is comonotonic. Extending this vector by including the sum of $-Z$ and $X+Z$ as a new component leads to $(-Z, X+Z, X)$, which is still comonotonic. Hence by Lemma $6, Z \in H(X)$.

\section{Proper hedge and convex order}

In this section, we show that both conjectures (4) and (5) hold true if $Z$ is a proper hedge of $X$.

Proposition 1 If $X \leq_{c x} Y$ and $Z \in H(X)$, then $X+Z \leq_{c x} Y+\mathbb{E}(Z)$. In particular, all proper hedges of $X$ are counter-monotonic convex hedges of $X$.

Proof: Fix any $p \in(0,1)$. Since $Z \in H(X),(-Z, X+Z)$ is comonotonic by Lemma 7 . It follows from Lemma 3 that

$$
\operatorname{TVaR}_{p}(X+Z)+\operatorname{TVaR}_{p}(-Z)=\operatorname{TVaR}_{p}(X) .
$$

Since $X \leq_{c x} Y$ and $-\mathbb{E}(Z) \leq_{c x}-Z$, Lemma 1 implies that

$$
\operatorname{TVaR}_{p}(X) \leq \operatorname{TVaR}_{p}(Y)
$$

and

$$
-\mathbb{E}(Z)=\operatorname{TVaR}_{p}(-\mathbb{E}(Z)) \leq \operatorname{TVaR}_{p}(-Z) .
$$

Combining all these, we have

$$
\operatorname{TVaR}_{p}(X+Z)=\operatorname{TVaR}_{p}(X)-\operatorname{TVaR}_{p}(-Z) \leq \operatorname{TVaR}_{p}(Y)+\mathbb{E}(Z)=\operatorname{TVaR}_{p}(Y+\mathbb{E}(Z)) .
$$

By Lemma 1 again, $X+Z \leq_{c x} Y+\mathbb{E}(Z)$. 
Proposition 2 If $X \leq_{c x} Y$ and $Z \in H(X)$, then $X+Z \leq_{c x} Y+Z$.

Proof: Similarly as done in the proof of Proposition 1, for any $0<p<1$, we have

$$
\operatorname{TVaR}_{p}(X+Z)=\operatorname{TVaR}_{p}(X)-\operatorname{TVaR}_{p}(-Z) \leq \operatorname{TVaR}_{p}(Y)-\operatorname{TVaR}_{p}(-Z) \leq \operatorname{TVaR}_{p}(Y+Z),
$$

where the last inequality follows from the subadditivity of TVaR. By Lemma $1, X+Z \leq_{c x} Y+Z$.

\section{Implications in optimal insurance problems}

Suppose that a risk bearer is exposed to a random positive insurable loss $X$, and wishes to reduce the risk by purchasing an insurance for $X$. Let $I$ be the indemnity purchased such that the risk bearer will receive $I(x)$ from the insurer if $x$ is the realized loss of $X$. In this section, we assume that the indemnity schedule $I$ is increasing and satisfies $0 \leq I(x) \leq x$. Severe moral hazard may result without these assumptions.

The insurer, relying on the law of large number, usually sets the premium $P$ at a level that is greater than $\mathbb{E}[I(X)]$ to avoid a loss on average. Suppose that the risk bearer is an expected utility maximizer with a wealth $w$ and an increasing and concave utility function $u$. He will be better off after purchasing $I$ if

$$
\mathbb{E} u(w-X+I(X)-P) \geq \mathbb{E} u(w-X) .
$$

Let $P^{*}$ be the amount of premium that makes the above inequality an equality. This is the maximum premium the risk bearer is willing to pay for $I$. In order for this insurance arrangement to be mutually beneficial to both the risk bearer and the insurer, $P^{*}$ has to be greater than $\mathbb{E}[I(X)]$. This leads us to the following definition:

Definition 3 Let $I$ be an indemnity schedule. If for any positive integrable random variable $X$, any wealth level $w$ and any increasing and concave utility function $u$, the solution to the equation

$$
\mathbb{E} u(w-X+I(X)-P)=\mathbb{E} u(w-X)
$$

is greater than $\mathbb{E}[I(X)]$, then we say $I$ is a universally marketable indemnity.

From this definition, an indemnity schedule $I$ is universally marketable if a mutually acceptable premium can be found for every risk bearer, regardless of the wealth, the utility function, and the underlying risk. This notion depends on how we judge the acceptability of a given premium. In our setting here, a premium is acceptable to the insurer if it is no less than the expected payment (so we are essentially assume the risk-neutrality of the insurer), and is acceptable to the risk bearer if a higher level of expected utility can be achieved after purchasing the insurance. 
It is evident that a given indemnity schedule $I$ may not be universally marketable without possessing some extra structures. The next result provides a simple sufficient condition for universal marketability.

Proposition 3 If an indemnity schedule I is 1-Lipschitz, i.e.

$$
0 \leq s \leq t \Longrightarrow I(t)-I(s) \leq t-s
$$

then it is universally marketable.

Proof: Let $X$ be an arbitrary positive integrable random variable. Suppose that $I$ is an 1-Lipschitz indemnity schedule. The 1-Lipschitzian property of $I$ implies that $x-I(x)$ is increasing in $x$, and thus $(X, X-I(X))$ is comonotonic. It then follows from Lemma 6 that $-I(X) \in H(X)$. Proposition 1 then implies

$$
X-I(X) \leq_{c x} X-\mathbb{E}[I(X)]
$$

Therefore, for any wealth level $w$ and any increasing and concave utility function $u$,

$$
\mathbb{E} u(w-X+I(X)-\mathbb{E}[I(X)]) \geq \mathbb{E} u(w-X)=\mathbb{E} u\left(w-X+I(X)-P^{*}\right) .
$$

As $u$ is increasing, $P^{*} \geq \mathbb{E}[I(X)]$.

In fact, most commonly encountered indemnity schedules are 1-Lipschitz, such as (i) quotashare contracts $I(x)=c x, c \in[0,1]$; (ii) deductible contracts $I(x)=(x-d)_{+}, d \geq 0$; (iii) limited loss contracts $I(x)=\max (x, a), a \geq 0$; (vi) insurance layer contracts $I(x)=(x-d)_{+}-(x-a)_{+}$, $a \geq d \geq 0$; and (v) contracts that are convex. Proposition 3 explains their popularity in the insurance market.

\section{Association and convex order}

Recall from (2) that the condition that $(Y, Z)$ is comonotonic guarantees the validity of

$$
X \leq_{c x} Y \quad \Longrightarrow \quad X+\mathbb{E}(Z) \leq_{c x} Y+Z .
$$

In this section, we show that the comonotonicity of $(Y, Z)$ can be weakened by using the notion of association.

Definition 4 A pair of random variables $\left(X_{1}, X_{2}\right)$ is said to be

(b) positively associated if $\operatorname{Cov}\left(f\left(X_{1}, X_{2}\right), g\left(X_{1}, X_{2}\right)\right) \geq 0$ for any increasing functions $f, g$ : $\mathbb{R}^{2} \rightarrow \mathbb{R}$ 
(b) weakly positively associated if $\operatorname{Cov}\left(f\left(X_{1}\right), g\left(X_{2}\right)\right) \geq 0$ for any increasing functions $f, g$ : $\mathbb{R} \rightarrow \mathbb{R}$

(c) negatively associated if $\operatorname{Cov}\left(f\left(X_{1}\right), g\left(X_{2}\right)\right) \leq 0$ for any increasing functions $f, g: \mathbb{R} \rightarrow \mathbb{R}$.

From the definition, a comonotonic pair of random variables is weakly positively associated, and a counter-monotonic pair of random variables is negatively associated.

In fact, weakly positive association is equivalent to a notion called positive quadrant dependence. A pair of random variables $\left(X_{1}, X_{2}\right)$ is said to be positively quadrant dependent (PQD) if

$$
F_{X_{1}, X_{2}}\left(x_{1}, x_{2}\right) \geq F_{X_{1}}\left(x_{1}\right) F_{X_{2}}\left(x_{2}\right) \quad \text { for all real numbers } x_{1}, x_{2} \text {. }
$$

A proof of this equivalence can be found, for innstance, in Dhaene and Goovaerts (1996). Similarly, negative association is equivalent to negative quadrant dependence, where $\left(X_{1}, X_{2}\right)$ is said to be negatively quadrant dependent (NQD) if

$$
F_{X_{1}, X_{2}}\left(x_{1}, x_{2}\right) \leq F_{X_{1}}\left(x_{1}\right) F_{X_{2}}\left(x_{2}\right) \quad \text { for all real numbers } x_{1}, x_{2} .
$$

We refer to Denuit et al. (2005), Mari and Kotz (2001), and Müller and Stoyan (2002) for a more detailed discussion of these notions.

The next result generalizes relation (2) by weakening the comonotonicity requirement on $(Y, Z)$ to $\mathrm{PQD}$.

Proposition 4 Suppose that $X_{i} \leq_{c x} Y_{i}$ for $i=1,2$. If $\left(X_{1}, X_{2}\right)$ is $N Q D$ and $\left(Y_{1}, Y_{2}\right)$ is $P Q D$, then $X_{1}+X_{2} \leq_{c x} Y_{1}+Y_{2}$.

Proof: Let $\left(X_{1}^{\perp}, X_{2}^{\perp}\right)$ and $\left(Y_{1}^{\perp}, Y_{2}^{\perp}\right)$ be copies of $\left(X_{1}, X_{2}\right)$ and $\left(Y_{1}, Y_{2}\right)$ respectively with independent components. $\left(X_{1}, X_{2}\right)$ being NQD implies that

$$
X_{1}+X_{2} \leq_{c x} X_{1}^{\perp}+X_{2}^{\perp},
$$

see, for example, Dhaene and Goovaerts (1996). On the other hand, $\left(Y_{1}, Y_{2}\right)$ being PQD implies that

$$
Y_{1}^{\perp}+Y_{2}^{\perp} \leq_{c x} Y_{1}+Y_{2}
$$

Since $X_{1}^{\perp}+X_{2}^{\perp} \leq_{c x} Y_{1}^{\perp}+Y_{2}^{\perp}$, the result follows.

Corollary 1 If $X \leq_{c x} Y$ and $(Y, Z)$ is weakly positively associated, then $X+\mathbb{E}(Z) \leq_{c x} Y+Z$.

This corollary extends a result in Bakhtin (2001) (also see p. 115 of Bulinski and Shashkin (2007)), in which the same conclusion is drawn under the stronger condition that $(Y, Z)$ is positively associated. 
Corollary 2 If $X \leq_{c x} Y,(X, Z)$ is $N Q D$ and $(Y, Z)$ is $P Q D$, then $X+Z \leq_{c x} Y+Z$.

When compared to Proposition 2, the counter-monotonicity of $(X, Z)$ is weakened to NQD in this corollary. On the other hand, a positive dependence structure (PDQ) on $(Y, Z)$ is needed here.

Upon examining the proof of Proposition 4 closely, one finds that the notions of PQD and NQD come into play only through comparisons with the independent sums $X_{1}^{\perp}+X_{2}^{\perp}$ and $Y_{1}^{\perp}+Y_{2}^{\perp}$. If we compare the distribution functions of $\left(X_{1}, X_{2}\right)$ and $\left(Y_{1}, Y_{2}\right)$ directly, we arrive at the following result:

Proposition 5 Suppose that $F_{X_{1}, X_{2}}(s, t) \leq F_{Y_{1}, Y_{2}}(s, t)$ for all real numbers $s, t$, and $\mathbb{E}\left(X_{i}\right) \leq$ $\mathbb{E}\left(Y_{i}\right)$ for $i=1,2$, then $X_{1}+X_{2} \leq_{s l} Y_{1}+Y_{2}$.

Proof: The result follows immediately from the identity

$$
\mathbb{E}\left[\left(X_{1}+X_{2}-d\right)_{+}\right]=\mathbb{E}\left[X_{1}\right]+\mathbb{E}\left[X_{2}\right]-d+\int_{-\infty}^{+\infty} F_{X_{1}, X_{2}}(x, d-x) d x,
$$

which can be found for example in Dhaene et al. (2002a).

\section{References}

[1] Bakhtin, Y.Y. 2001. Limit theorems for the solutions of the stochastic Burgers equation. $\mathrm{PhD}$ thesis, Moscow.

[2] Björk, T., 2009. Arbitrage Theory in Continuous Time, 3rd Edition, Oxford University Press.

[3] Bulinski, A., Shashkin, A., 2007. Limit Theorems For Associated Random Fields And Related Systems. World Scientific, Singapore.

[4] Cheung, K.C., 2010. Characterizing comonotonic random vector by the distribution of the sum of its components. Insurance: Mathematics and Economics 47, 130-136.

[5] Denuit, M., Dhaene, J., Goovaerts, M.J., Kaas, R., 2005. Actuarial Theory for Dependent Risks. Wiley.

[6] Dhaene, J., Denuit, M., Goovaerts, M.J., Kaas, R., Vyncke, D., 2002a. The concept of comonotonicity in actuarial science and finance: applications. Insurance: Mathematics and Economics 31, 3-33. 
[7] Dhaene, J., Denuit, M., Goovaerts, M.J., Kaas, R., Vyncke, D., 2002b. The concept of comonotonicity in actuarial science and finance: applications. Insurance: Mathematics and Economics 31, 133-161.

[8] Dhaene, J., Goovaerts, M.J., 1996. Dependency of risks and stop-loss order. Astin Bulletin 26, 201-212.

[9] Dhaene, J., Vanduffel, S., Goovaerts, M.J., Kaas, R., Tang, Q., Vyncke, D., 2006. Risk measures and comonotonicity: a review. Stochastic Models 22, 573-606.

[10] Kallenberg, O., 2002. Foundations of Modern Probability (Second Edition). SpringerVerlag, New York.

[11] Mari, D.D., Kotz, S., 2001. Correlation and Dependence. Imperial College Press, London.

[12] Müller, A.; Stoyan, D., 2002. Comparison Methods for Stochastic Models and Risks. John Wiley \& Sons, Ltd., Chichester.

[13] Shaked, M., Shanthikumar, J.G., 1994. Stochastic Orders and Their Applications. Academic Press. 